\title{
The Royal College of Radiologists of Thailand in collaboration with Thailand Center of Excellence in Life Sciences shape the future of artificial intelligence in diagnostic radiology
}

Sitthichok Chaichulee, Ph.D. ${ }^{(1)}$

Thammasin Ingviya, M.D., Ph.D. ${ }^{(2)}$

Supharerk Thawillarp, M.D., D.P.H. ${ }^{(3)}$

Pattarawin Attasara, M.D. ${ }^{(4)}$

Wiwatana Tanomkiat, M.D. ${ }^{(5)}$

From ${ }^{(1)}$ Department of Biomedical Sciences and Biomedical Engineering,

${ }^{(2)}$ Department of Family and Preventive Medicine,

${ }^{(5)}$ Department of Radiology, Faculty of Medicine, Prince of Songkla University,

Hat Yai, Songkhla, 90110, Thailand.

(3) Epidemic Intelligence Unit (EIU), Department of Disease Control, Ministry of Public Health, Nonthaburi, 11000, Thailand.

(4) Digital Medical Bureau, Medical Services Department, Ministry of Public Health, Nonthaburi, 11000, Thailand.

Address correspondence to W.T. (email: twiwadha@hotmail.com)

Received 21 April 2021; accepted 25 April 2021

doi:10.46475/aseanjr.v22i1.126

Keywords: Artificial intelligence, AI, Diagnostic Radiology, Thailand.

The COVID-19 pandemic has disrupted the traditional way of living in almost all aspects. Physical distancing was suggested, and partial lock-down policies have been implemented. The development and uses of technology are being accelerated to overcome the massive obstacle for humankind, or even to eliminate this fatal infection. 
Before the pandemic, tuberculosis (TB) has long been a major health problem in Thailand throughout its 800 years of history or longer. Currently, Thailand has been classified by the World Health Organization (WHO) as one of the 22 countries in the world with the highest TB burden. As the number one cause of death amongst infectious diseases at the global level, more prominent than AIDS, the United Nation (UN) and WHO have listed TB control as part of their Sustainable Development Goals (SDGs). With the TB incidence of 1.3 times the global rate, Thailand is committed to decrease new cases to less than 10 per 100 000 population within 2025 [1]. To achieve this highly challenging goal, all actively infected cases will have to be found and treated. Since TB commonly attacks persons who are usually in a weakened condition or living in a confined space, Thailand's case-finding strategy is currently applied with 7 high-risk groups including persons who have contact with patients diagnosed with TB, HIVinfected patients, diabetic patients with poor glycemic control, aged persons with chronic diseases, prisoners, migrant workers, and health care providers. Because of its high sensitivity, the chest radiograph is used as a screening tool for these 7 high-risk groups, regardless of their symptoms. The screening campaign has included the prisoners in 2017. The people in the other 6 high-risk groups will be covered by 2021. A rapid increase in the uses of chest radiographs is expected. The interpretation of the large volume of chest radiographs will be a heavy burden to radiologists. In addition, since the interpretation by radiologists is not timely made, patients will probably be detected in a delayed manner, especially when their chest radiographs are abnormal. AI is expected to triage and screen the normal films at the site.

Several AIs have been developed for reading chest radiographs. They are either imported or locally invented. While AI seems to be a good prospect in assisting the medical team, there remain many questions to be answered. The sensitivity of disease detections, the experience and feeling of the patients and the service providers, safety and security of patients' data, changes that might disrupt the hospital workflow and the structure of the radiology unit, the sustainability and fairness to the buyers and more, are some examples. Doubts in the usefulness and adverse reactions of AIs will lead to reluctance in the development of new AI algorithms. 
To support the AI users, the Royal College of Radiologists of Thailand (RCRT) has developed the AI user guideline covering all essential points to help users make an informed decision regarding the purchase and uses of AI products. The guideline could help the users understand the problem they are trying to solve, assess whether the AI product is an appropriate solution for their organization, interpret the performance claims made by the vendor, assure whether the product meets regulatory, safety and ethical standards, ensure whether the processing of data fully complies with the organizational data privacy policy, thus, promoting the sustainable use of $\mathrm{AI}$ in the organization. It is believed that this guideline will increase the confidence in both users in purchasing the products and inventors and vendors in providing services and products to healthcare providers.

TCELS, under the supervision of the Ministry of Higher Education, Science, Research and Innovation, is a public organization to promote research and innovation in healthcare. TCELS forms networks and shares the resources with many organizations in public, private and non-profit sections. The press release and conference of the collaboration between the 2 organizations and the introduction of AI user guideline was held on April 7, 2021 at Vie Hotel, Bangkok among TCELS alliance and RCRT board. 


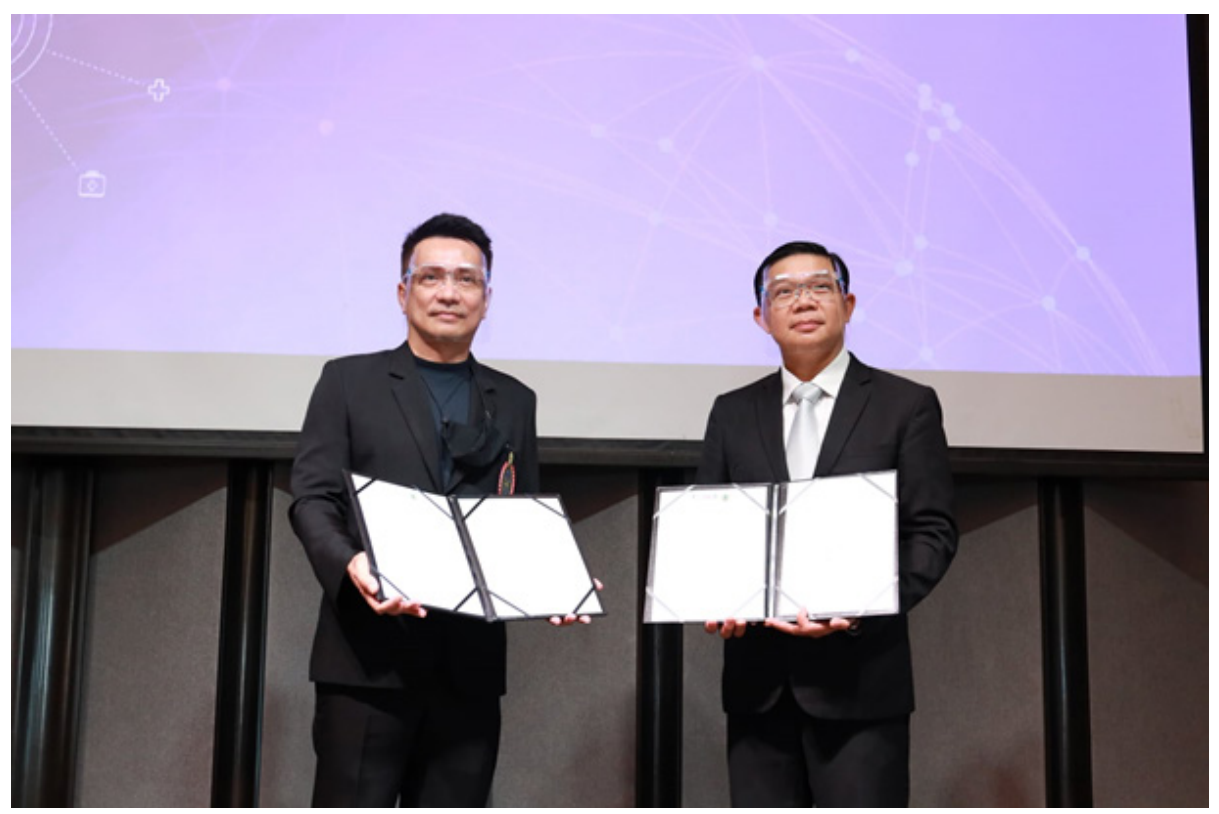

Figure 1. Mr.Sirasak Teparkum, the TCELS chief executive officer and Associate Professor Wiwatana Tanomkiat, the RCRT president show the memoirs of understanding in collaboration at the press conference.

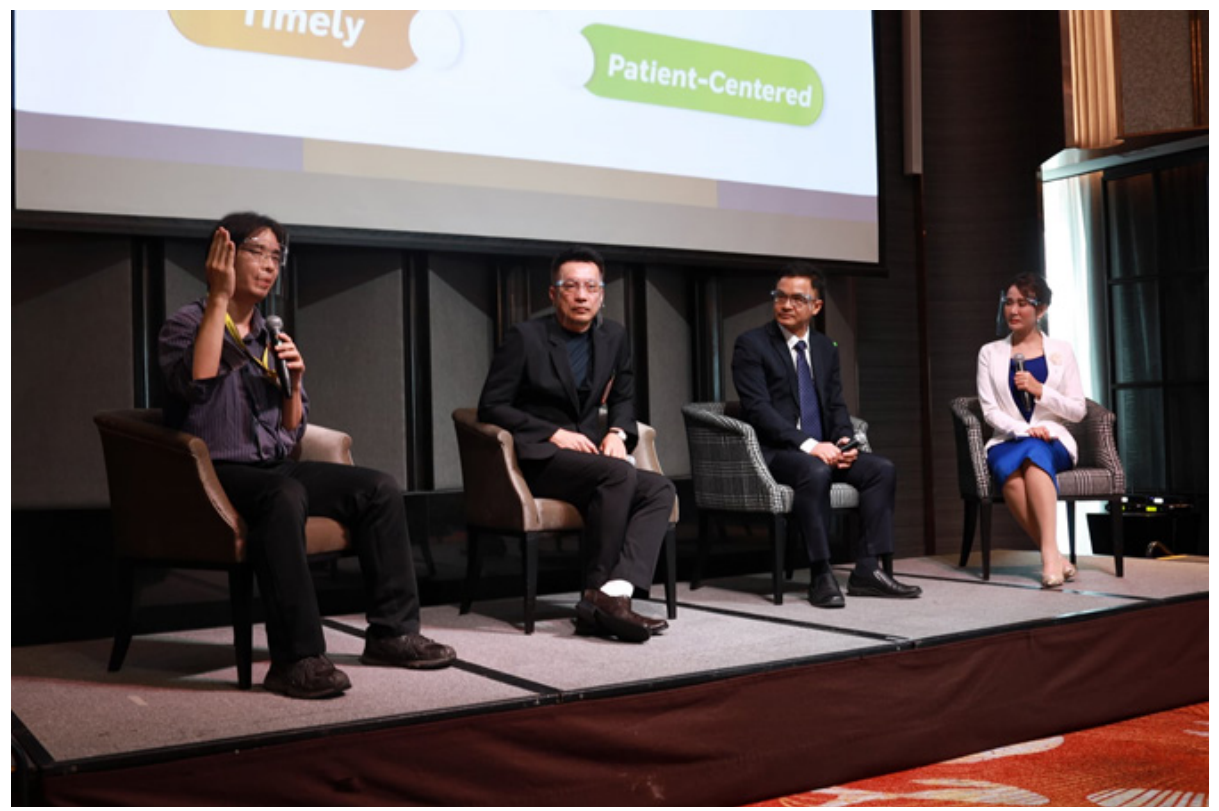

Figure 2. Dr. Supharerk Thawillarp as the representative of the RCRT board, explains how the guideline is developed to the audience in the press conference. 


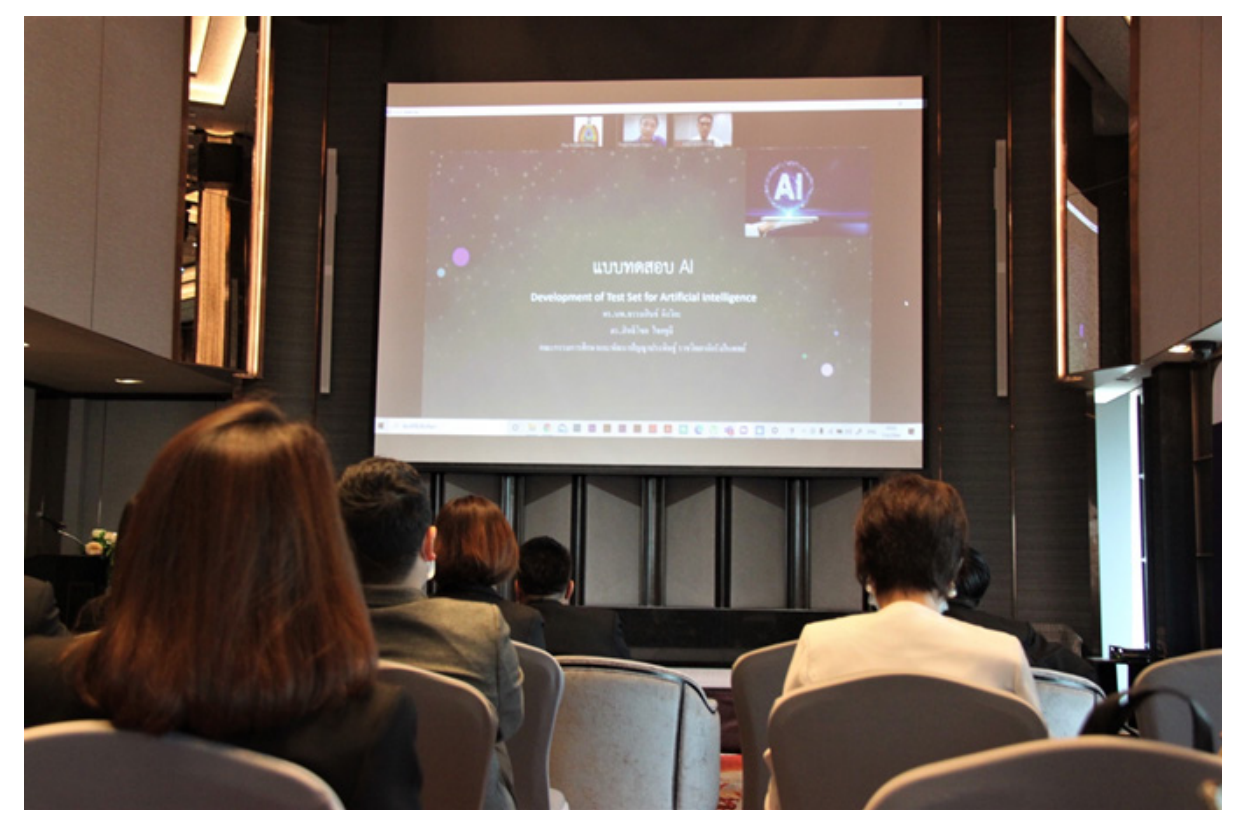

Figure 3. Dr.Thammasin Ingviya and Dr.Sitthichok Chaichulee as the representative of RCRT board give a virtual talk about the plan to develop standard sets of radiographs to test AI.

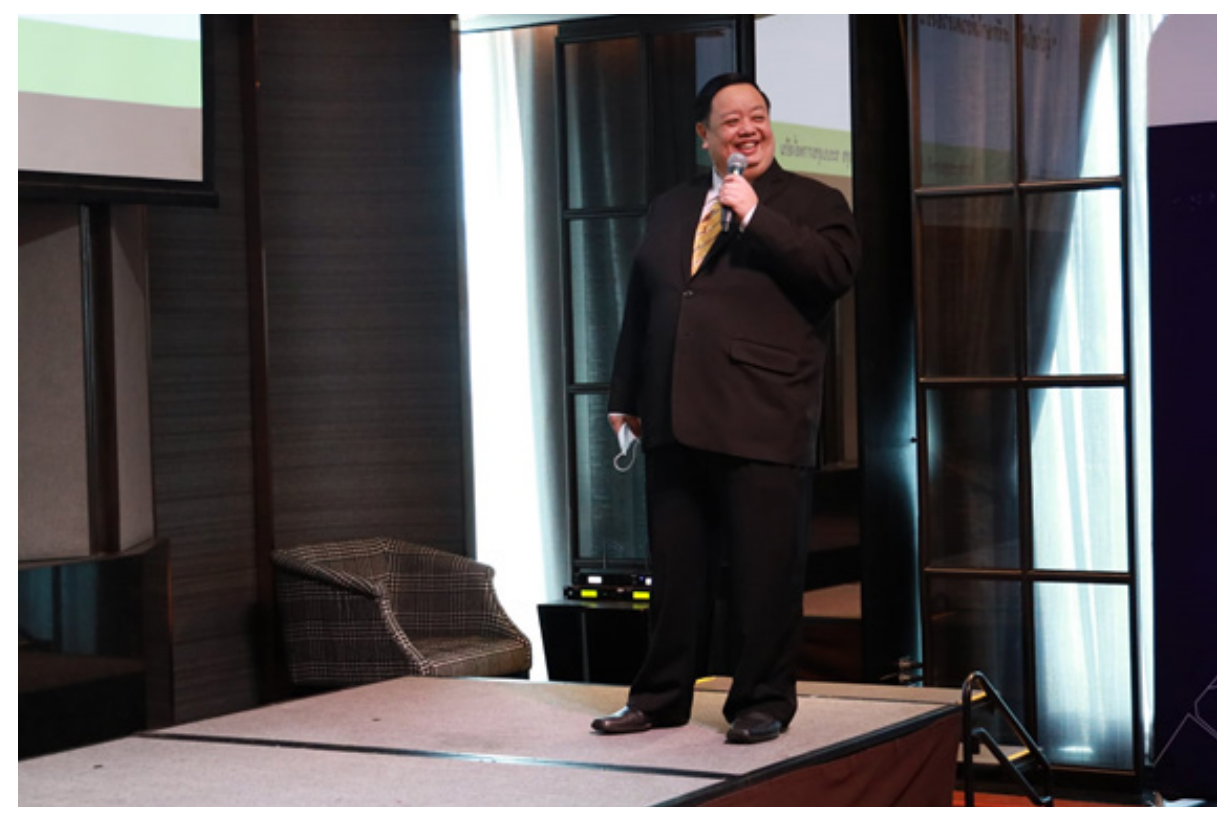

Figure 4. Dr.Pattarawin Attasara as a representative of RCRT board explains the current situation of tuberculosis, the development and use of AI in public health services, and the national policy to end tuberculosis. 
Our next step is to develop a test dataset of Thai chest radiographs, each of which is accurately labelled according to ILO (International Labour Office) classification by B readers who are rigorously certified by the National Institute for Occupational Safety and Health (NIOSH) and specialize in the interpretation of chest radiography. This dataset could serve as a reference for the testing of AI products. 


\section{References}

1. Bureau of Tubercolosis. Thailand oparational plan to end tuberculosis 20172021 [Internet]. Nonthaburi: The Bureau; 2017 [cited 2021 Apr 21]. Available from: https://www.tbthailand.org/download/Manual/Thailand\%20Operational \%20Plan\%20To\%20End\%20\%20TB_2017_2021.pdf 